ORNL/TM--12248

DE93 007788

Fusion Energy Division

\title{
AN OUTGOING ENERGY FLUX BOUNDARY CONDITION FOR FINITE DIFFERENCE ICRF ANTENNA MODELS
}

D. B. Batchelor

M. D. Carter

Date published: November 1992

\author{
Prepared by \\ OAK RIDGE NATIONAL LABORATORY \\ Oak Ridge, Tennessee 37831-6285 \\ managed by \\ MARTIN MARIETTA ENERGY SYSTEMS, INC. \\ for the \\ U.S. DEPARTMENT OF ENERGY \\ under contract DE-AC05-84OR21400
}




\section{CONTENTS}

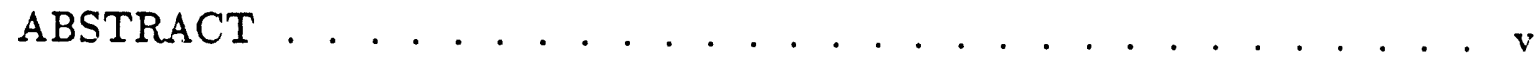

1. INTRODUCTION . . . . . . . . . . . . . . . . 1

2. DERIVATION OF THE BOUNDARY CONDITION . . . . . . . . 2

3. APPLICATION TO A SIMPLE 2D PROBLEM . . . . . . . . . . . 8

4. RESULTS OF 2D CALCULATION AND DISCUSSION . . . . . . . . 11 ACKNOWLEDGMENT . . . . . . . . . . . . . . . . 15

REFERENCES . . . . . . . . . . . . . . . . . 16 


\begin{abstract}
For antennas at the ion cyclotron range of frequencies (ICRF) modeling in vacuum can now be carried out to a high level of detail such that shaping of the current straps, isolating septa, and discrete Faraday shield structures can be included. An efficient approach would be to solve for the fields in the vacuum region near the antenna in three dimensions by finite methods and to match this solution at the plasma-vacuum interface to a solution obtained in the plasma region in one dimension by Fourier methods. This approach has been difficult to carry out because boundary conditions must be imposed at the edge of the finite difference grid on a point-by-point basis, whereas the condition for outgoing energy flux into the plasma is known only in terms of the Fourier transform of the plasma fields. A technique is presented by which a boundary condition can be imposed on the computational grid of a three-dimensional finite difference, or finite element, code by constraining the discrete Fourier transform of the fields at the boundary points to satisfy an outgoing energy flux condition appropriate for the plasma. The boundary condition at a specific grid point appears as a coupling to other grid points on the boundary, with weighting determined by a kernel calculated from the plasma surface impedance matrix for the various plasma Fourier modes. This boundary condition has been implemented in a finite difference solution of a simple problem in two dimensions, which can also be solved directly by Fourier transformation. Results are presented, and it is shown that the proposed boundary condition does enforce outgoing energy flux and yields the same solution as is obtained by Fourier methods.
\end{abstract}




\section{INTRODUCTION}

Ion cyclotron range of frequencies (ICRF) antennas are complicated, threedimensional (3D) structures whose performance can be affected by details of the geometry. The modeling of such antennas in vacuum is now sophisticated enough that minute details of the structure can be treated, including curved current straps, isolating septa, and discrete Faraday shield structures. This is typically done using finite difference or finite element computer codes (KRESS et al., 1991; RYAN et al., 1990). The presence of the plasma near the antenna is a critical feature, since induced currents in the antenna structure influence the spectrum of power radiated into the plasma. In turn, calculations with two-dimensional (2D) models (BATCHELOR et al., 1992) have shown that radio-frequency (RF) plasma currents induce their own image currents in the antenna, which can cause significant discrepancies between the loading and radiated spectrum calculated from self-consistent antenna/wall currents and the values predicted from vacuum measurements and calculations. It is generally not computationally feasible to include the plasma region in a 3D finite difference code because of the large plasma volume relative to the wavelength and the complexity of the plasma constitutive relation. In any case, from the antenna coupling standpoint, the plasma is essentially one-dimensional (only radial variation is important), so that 3D calculation of the RF fields inside the plasma by finite methods is an unnecessary computational burden.

A logical approach would be to solve for the fields in three dimensions near the antenna and to match this solution to a plasma solution which, because of the lower dimensionality of the plasma, can be obtained by Fourier methods. It has been difficult to carry out such a program because boundary conditions must be imposed at the edge of the finite difference grid on a point-by-point basis, whereas the condition for outgoing energy flux into the plasma is known only in terms of the Fourier transform of the fields in the plasma. We have now developed a technique by which a boundary condition can be imposed on the computational grid by constraining the discrete Fourier transform of the fields at the boundary points to satisfy an outgoing energy flux condition appropriate for the plasma. The boundary condition at a specific grid point appears as a coupling to other grid points on the boundary, with weighting determined by 
a kernel calculated from the plasma surface impedance matrix for the various Fourier modes.

In Section 2 we give a derivation of the method for coupling a $3 \mathrm{D}$ finite difference solution in a vacuum region to Fourier representations of the field solution in a semi-infinite slab plasma. In order to verify that this scheme does indeed impose the expected boundary condition, we have implemented a $2 \mathrm{D}$ finite difference model and applied it to the case of a simple current strap over a conducting ground plane, radiating into a slab plasma-a problem for which a solution is also available using Fourier methods. Section 3 describes the 2D model. Section 4 gives results of the finite difference model and compares them to the solution in terms of Fourier transforms.

\section{DERIVATION OF THE BOUNDARY CONDITION}

We consider the situation illustrated in Fig. 1. The region $x<0$ is vacuum containing an arbitrary $3 \mathrm{D}$ antenna structure. The computational domain extends over poloidal length $0 \leq y \leq a$ and toroidal length $0 \leq z \leq b$. Wr assume the fields to be periodic in the $y$ (poloidal) and $z$ (toroidal) directions. It is not necessary that $a$ extend to $2 \pi R_{\min }$ or that $b$ extend to $2 \pi R_{T}\left(R_{\min }=\right.$ minor radius, $R_{T}=$ major radius) in order to adequately model the antenna in cases for which the fields fall rapidly to zero with distance from the antenna. A magnetized plasma exists in the region $x \geq 0$, which is uniform in the poloidal and toroidal directions, $n_{s}(\mathbf{x})=n_{s}(x), \mathbf{B}_{0}(x)=B_{0}(x) \hat{z}$, where $n_{s}$ is the particle density of species $s$ and $\mathbf{B}_{0}$ is the equilibrium magnetic field.

The field in the vacuum region is presumed to be obtained from a finite difference or finite element solution of Maxwell's equations in three dimensions,

$$
\begin{aligned}
& \nabla \times \mathbf{E}=i \omega \mathbf{B}, \\
& \nabla \times \mathbf{B}=-i \omega \mathbf{E}-i \omega \mu_{0} \mathbf{J}_{\mathbf{e x t}},
\end{aligned}
$$

subject to appropriate boundary conditions on the metal structure, periodic boundary conditions at $z=0, b$ and at $y=0, a$, and conditions of outgoing energy flow at the plasma-vacuum interface, $x=0$. The task at hand then is to impose such outgoing energy boundary conditions on the finite difference solution in terms of the Fourier representation of the field in the plasma region. 


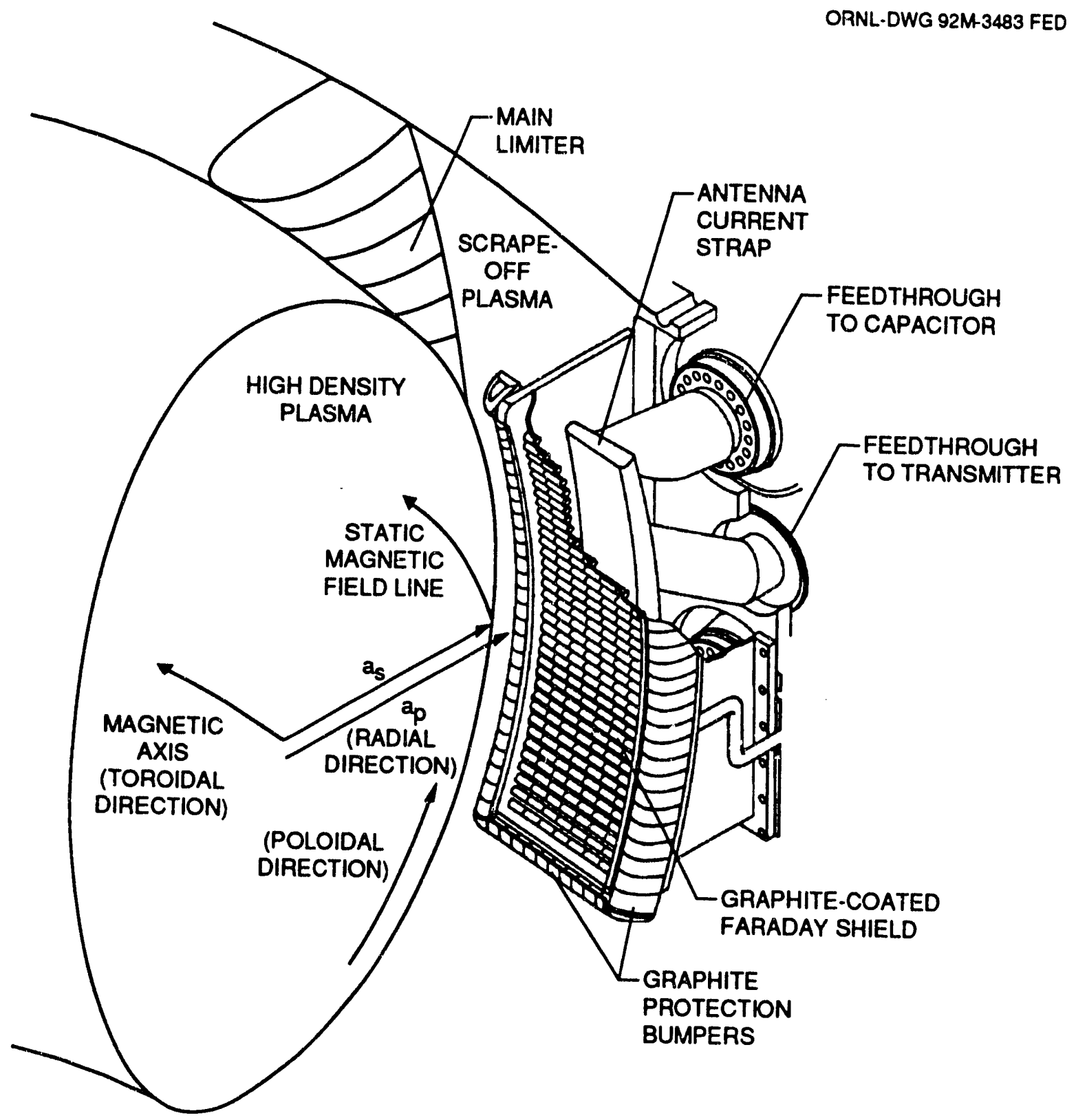

Fig. 1. Geometry of 3D antenna structure radiating into slab plasma having variation only in the $x$-direction. 
This boundary condition involves imposing the proper linear relation between the wave electric field and magnetic field (or equivalently between the wave electric field and its derivatives) at the edge of the computational grid. Precisely which field components are needed depends on details of the numerical implementation. For concreteness we assume that $\mathbf{B}_{\mathbf{T}}$, the tangential components of $\mathbf{B}$, are to be expressed in terms of $\mathbf{E}_{\mathbf{T}}$, the tangential components of $\mathbf{E}$.

In the plasma region the fields satisfy a wave equation of the form

$$
\begin{aligned}
& \nabla \times \mathbf{E}=i \omega \mathbf{B}, \\
& \nabla \times \mathbf{B}=-i \omega \mathbf{E}-i \omega \mu_{0} \mathbf{J}_{\mathbf{P}},
\end{aligned}
$$

where $\mathbf{J}_{\mathbf{P}}$ is the wave-induced plasma current. Typically we assume $\mathbf{J}_{\mathbf{P}}$ to be of the form $\mathbf{J}_{\mathbf{P}}=\Sigma \cdot \mathbf{E}$, where $\Sigma$ is the cold or warm plasma conductivity tensor. Nothing in the formulation, however, prevents $\mathbf{J}_{\mathbf{P}}$ from being a more general operator, such as an integral operator that requires no small Larmor radius expansion. In view of the uniformity of the plasma in the $y$-and $z$-directions, $\mathbf{E}$ and $\mathbf{B}$ can be expanded in a Fourier series,

$$
\mathbf{E}(\mathbf{x})=\sum_{m n \sigma} A_{\sigma}^{m n} \mathbf{E}_{\sigma}^{m n}(x) e^{2 \pi i(m y / a+n z / b)}
$$

where $\sigma=1,2$ indicates the two independent polarizations at the plasma edge. Equation (2) then becomes a set of ordinary differential equations for each value of $m, n, \sigma$. For given $m, n$ there are in general several modes that propagate in the plasma, for example fast and slow cyclotron waves and, in a warm plasma, Bernstein waves. When a warm plasma is treated the temperature profile is taken to go smoothly to zero at the plasma boundary, so that the solutions are determined uniquely from the tangential components of $\mathbf{E}$ (or $\mathbf{B}$ ) at the plasma boundary. Bernstein waves, if present, are excited by the fields penetrating into the plasma. The zero temperature boundary condition then ensures that Bernstein waves propagating in the direction of negative $x$ are totally reflected inside the plasma.

Equations (2) are solved for each $m, n, \sigma$, imposing on each solution a boundary condition of outgoing energy flux at large $x$ and an initial magnetic field polarization at $x=0$,

$$
\mathbf{b}_{\sigma}^{m n} \equiv \mathbf{B}_{\mathbf{T}}(x=0)^{m n}=b_{y \sigma}^{m n} \hat{y}+b_{z \sigma}^{m n} \hat{z}
$$


A convenient choice for the polarization eigenvectors is $\left(b_{y \sigma}^{m n}=1, b_{z \sigma}^{m n}=0\right)$ for $\sigma=1$ (slow wave excitation), $\left(b_{y \sigma}^{m n}=0, b_{z \sigma}^{m n}=1\right)$ for $\sigma=2$ (fast wave excitation), although such a choice is not required. By this process we obtain the plasma eigenfunctions $\mathrm{E}_{\sigma}^{m n}(x)$ of $\mathrm{Eq}$. (3) with the $A_{\sigma}^{m n}$ representing coefficients to be determined.

Evaluating $\mathbf{E}_{\sigma}^{m n}(x=0)$, we can construct an effective plasma surface impedance matrix $\mathbf{Z}^{m n}$ which expresses for the $m, n$ Fourier mode the tangential electric field at the plasma-vacuum boundary in terms of the tangential magnetic field, $\mathbf{E}_{\mathbf{T}}^{m n}=\mathbf{Z}^{m n} \cdot \mathbf{B}_{\mathbf{T}}^{m n}$. Introducing the $2 \mathrm{D}$ electric field polarization eigenvectors,

$$
\mathbf{e}_{\sigma}^{m n} \equiv \mathbf{E}_{\mathbf{T} \sigma}^{m n}(x=0)=E_{y \sigma}^{m n} \hat{y}+E_{z \sigma}^{m n} \hat{z},
$$

we have, in particular,

$$
\mathbf{e}_{\sigma}^{m n}=\mathbf{Z}^{m n} \cdot \mathbf{b}_{\sigma}^{m n}
$$

so the tangential part of Eq. (3), evaluated at $x=0$, can be expressed as

$$
\mathbf{E}_{\mathbf{T}}(0, y, z)=\sum_{m n \sigma} A_{\sigma}^{m n} \mathbf{e}_{\sigma}^{m n} e^{2 \pi i(m y / a+n z / b)}=\sum_{m n \sigma} A_{\sigma}^{m n} \mathbf{Z}^{m n} \cdot \mathbf{b}_{\sigma}^{m n} e^{2 \pi i(m y / a+n z / b)},
$$

whereas

$$
\mathbf{B}_{\mathbf{T}}(0, y, z)=\sum_{m n \sigma} A_{\sigma}^{m n} \mathbf{b}_{\sigma}^{m n} e^{2 \pi i(m y / a+n z / b)}
$$

Fourier transforming Eq. (4) with respect to $y$ and $z$, we obtain

$$
\sum_{\sigma} A_{\sigma}^{m n} \mathbf{e}_{\sigma}^{m n}=\frac{1}{a b} \int d y d z \mathbf{E}_{\mathbf{T}}(0, y, z) e^{-2 \pi i(m y / a+n z / b)} .
$$

Since the $\mathbf{e}_{\sigma}^{m n}, \sigma=1,2$ are linearly independent, we can construct a set of adjoint eigenvectors $\mathbf{e}_{\sigma}^{\dagger m n}$ such that

$$
\mathbf{e}_{\sigma}^{\dagger m n} \cdot \mathbf{e}_{\sigma^{\prime}}^{m n}=\delta_{\sigma \sigma^{\prime}}
$$

For example, if one chooses for the magnetic field polarization vectors $\mathbf{b}_{\sigma=1}^{m n}=$ $(1,0)$ and $\mathbf{b}_{\sigma=2}^{m n}=(0,1)$, the adjoint eigenvectors are simply given by

$$
\mathbf{e}_{\sigma}^{\dagger m n}=\left[Z^{m n}\right]^{-1} \cdot \mathbf{e}_{\sigma}^{m n}
$$

Using these, Eq. (6) can be solved for the $A_{\sigma}^{m n}$ to give

$$
A_{\sigma}^{m n}=\frac{1}{a b} \int d y d z \mathbf{e}_{\sigma}^{\dagger m n} \cdot \mathbf{E}_{\mathrm{T}}(0, y, z) e^{-2 \pi i(m y / a+n z / b)}
$$


Substituting Eq. (8) in Eq. (5) gives

$$
\begin{aligned}
\mathbf{B}_{\mathbf{T}}(0, y, z)= & \sum_{m n \sigma} \mathbf{b}_{\sigma}^{m n} e^{2 \pi i(m y / a+n z / b)} \\
& \times \frac{1}{a b} \int d y^{\prime} d z^{\prime} \mathbf{e}_{\sigma}^{\dagger m n} \cdot \mathbf{E}_{\mathbf{T}}\left(0, y^{\prime}, z^{\prime}\right) e^{-2 \pi i\left(m y^{\prime} / a+n z^{\prime} / b\right)} \\
= & \int d y^{\prime} d x^{\prime} \mathbf{H}\left(y-y^{\prime}, z-z^{\prime}\right) \cdot \mathbf{E}_{\mathbf{T}}(0, y, z),
\end{aligned}
$$

where the dyadic kernel $\mathbf{H}\left(y-y^{\prime}, z-z^{\prime}\right)$ is given by

$$
\mathbf{H}\left(y-y^{\prime}, z-z^{\prime}\right)=\sum_{m n \sigma} \mathbf{b}_{\sigma}^{m n} \mathbf{e}_{\sigma}^{\dagger m n} e^{2 \pi i\left[m\left(y-y^{\prime}\right) / a+n\left(z-z^{\prime}\right) / b\right]} .
$$

Equation (9) constitutes the desired condition, which enforces outgoing energy flux into the plasma. Since our interest here is to impose this boundary condition on a finite solution in the vacuum region, Eq. (9) must be discretized in some manner. Again, the precise way in which this is to be done depends on the details of implementation of the numerical finite solution, for example, on whether the solution is by finite differences or finite elements. For concreteness we consider a finite difference solution such that the field quantities are defined on a grid of mesh points $\left(x_{i}, y_{j}, z_{k}\right)$. The integral in Eq. (9) is obtained from a quadrature rule of the form

$$
\mathbf{B}_{\mathbf{T}}\left(0, y_{i}, z_{j}\right)=\sum_{l m} \mathbf{W}_{i j ; l m} \cdot \mathbf{E}_{\mathbf{T}}\left(0, y_{l}, z_{m}\right),
$$

where $\mathbf{W}_{i j ; l m}$ are the weights of the quadrature rule.

In the special but important case of grid points uniformly spaced in $y$ and $z$, one can dispense with the integral quadrature altogether and represent the fields at the grid points directly in terms of discrete Fourier transforms. Denote the grid points on the plasma-vacuum boundary as $y_{i}=i a / N_{y}, 0 \leq i \leq N_{y}$ and $z_{j}=j b / N_{z}, 0 \leq j \leq N_{z}$, where $N_{y}$ and $N_{z}$ are the number of grid points in each direction. Then Eq. (4) can be expressed as

$$
\mathbf{E}_{\mathrm{T}}\left(0, y_{i}, z_{j}\right)=\sum_{m n \sigma} A_{\sigma}^{m n} \mathbf{e}_{\sigma}^{m n} e^{2 \pi i\left(m i / N_{y}+n j / N_{z}\right)}
$$


and similarly for Eq. (5). Taking a discrete Fourier transform of this equation gives

$$
\begin{aligned}
\sum_{\sigma} A_{\sigma}^{m n} \mathrm{e}_{\sigma}^{m n} & =\frac{1}{N_{y} N_{z}} \sum_{i=0, j=0}^{N_{y}, N_{z}} \mathbf{E}_{\mathbf{T}}\left(0, y_{i}, z_{j}\right) e^{-2 \pi i\left(m i / N_{y}+n j / N_{z}\right)}, \\
& -\frac{N_{y}}{2} \leq m \leq \frac{N_{y}}{2}, \quad-\frac{N_{z}}{2} \leq n \leq \frac{N_{z}}{2}
\end{aligned}
$$

From the Nyquist sampling theorem we know that $N+1$ sample points will serve to define $N+1$ Fourier harmonics, which we take in Eq. $\left(6^{\prime}\right)$ to lie in the range $-\left(N_{y} / 2\right) \leq m \leq\left(N_{y} / 2\right),-\left(N_{z} / 2\right) \leq n \leq\left(N_{z} / 2\right)$. Equation $\left(6^{\prime}\right)$ can be solved for the $A_{\sigma}^{m n}$ using the adjoint eigenvectors, as Eq. (8) was. When these coefficients are used in the discrete Fourier representation for $\mathbf{B}_{\mathbf{T}}(0, y, z)$, one obtains an equivalent of Eq. (9),

$$
\mathbf{B}_{\mathbf{T}}\left(0, y_{i}, z_{j}\right)=\sum_{l, k=0}^{N_{y}, N_{\mathbf{z}}} \mathbf{H}\left(y_{i}-y_{l}^{\prime}, z_{j}-z_{k}^{\prime}\right) \cdot \mathbf{E}_{\mathbf{T}}\left(0, y_{l}, z_{k}\right),
$$

where

$$
\mathbf{H}\left(y_{i}-y_{l}^{\prime}, z_{j}-z_{k}^{\prime}\right)=\sum_{m=-\frac{N_{y}}{2}, n=-\frac{N_{x}}{2}, \sigma}^{\frac{N_{y}}{2}, \frac{N_{z}}{2}} \mathbf{B}_{\sigma}^{m n} \mathbf{e}_{\sigma}^{\dagger m n} e^{i\left[m(i-l) / N_{y}+n(j-k) / N_{x}\right]} .
$$

This is precisely what would be obtained from Eq. (11) in the case of equally spaced grid points where the quadrature formula is the simple trapezoidal rule with increments $h_{y}=a / N_{y}$ and $h_{z}=b / N_{z}$. Then the weights $W_{i j ; l m}$ are simply

$$
\mathbf{W}_{i j ; l m}=h_{y} h_{z} \mathbf{H}\left(y_{i}-y_{l}, z_{j}-z_{m}\right) \text {. }
$$

Equation (11) [or Eq. (9') for the case of a uniform grid] constitutes the desired discretized boundary condition on $\mathbf{B}_{\mathbf{T}}$ in terms of the values of $\mathbf{E}_{\mathbf{T}}$ evaluated at the grid points. 


\section{APPLICATION TO A SIMPLE 2D PROBLEM}

\subsection{Reduction of the Boundary Condition to Fast Wave}

\section{Propagation in Two Dimensions}

In order to verify that this scheme does indeed enforce the expected condition on energy flow, we have developed a finite difference solution of the simple 2D problem sketched in Fig. 2. The plasma region, $x>0$, is again uniform in $y$ and $z$. A sheet, $y$-directed current distribution is present at $x=-x_{A}$,

$$
\mathrm{J}_{\mathrm{ext}}(x, z)=J_{y}(z) \delta\left(x+x_{A}\right) \hat{y}
$$

and a flat conducting wall is located at $x=-x_{w}$. Further, we restrict consideration to the TE mode of propagation, or fast wave, for which $\mathbf{E}(x, z)=$ $E_{y}(x, z) \hat{y}$ and $\mathbf{B}(x, z)=B_{x}(x, z) \hat{x}+B_{z}(x, z) \hat{z}$. In this case, it is convenient to eliminate $\mathbf{B}$ using Faraday's law,

$$
B_{z}=\frac{1}{i \omega} \frac{\partial E_{y}}{\partial x}, \quad B_{x}=\frac{-1}{i \omega} \frac{\partial E_{y}}{\partial z} .
$$

We then work with the second-order equation for $E_{y}$ alone,

$$
\nabla^{2} E_{y}+\frac{\omega^{2}}{c^{2}} E_{y}=-i \omega J_{y}
$$

where $\nabla^{2}=\left(\partial^{2} / \partial x^{2}\right)+\left(\partial^{2} / \partial z^{2}\right)$.

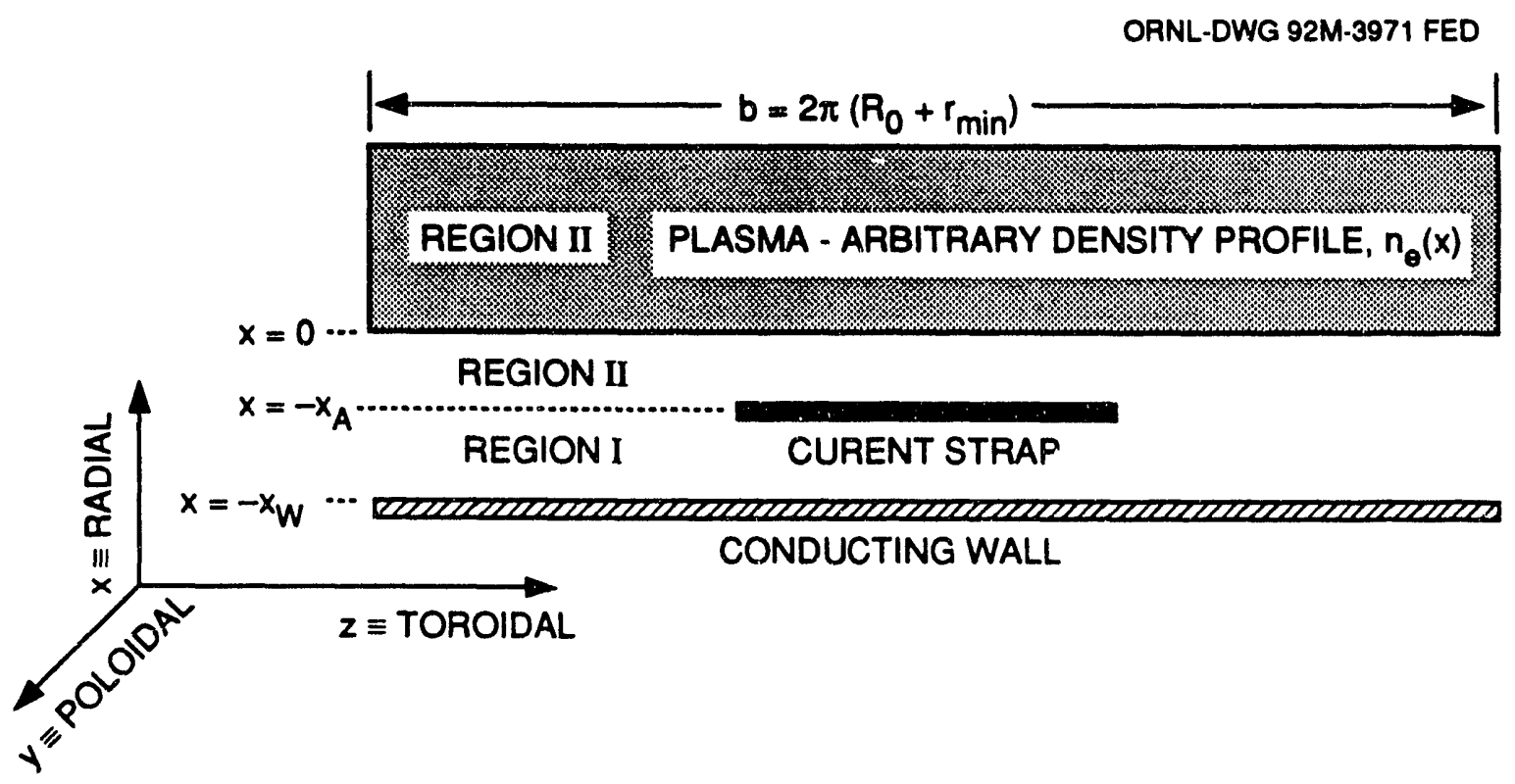

Fig. 2. Geometry for 2D problem. 
In the plasma region we can write $E_{y}(x, z)$ as

$$
E_{y}(x, z)=\sum_{n} A_{n} E^{n}(x) e^{i n K z},
$$

where $K=2 \pi / b, E^{n}(x)$ is the outgoing energy solution of the ordinary differential equation

$$
\left[\frac{\partial^{2}}{\partial x^{2}}+k_{x}^{2}(x, n)\right] E^{n}(x)=0,
$$

and $k_{x}^{2}(x, n)$ is the fast wave solution of the plasma dispersion relation $D\left(\omega, x, k_{x}, k_{z}=n K\right)=0$.

For the finite difference solution of Eq. (15) we will require a boundary condition relating $E_{y}^{\prime}(x, z)$ [or equivalently $B_{z}(x, z)$ ] to $E_{y}(x, z)$. Here the prime refers to differentiation by $x$. Differentiating Eq. (16) gives

$$
\left.E_{y}^{\prime}(x, z)=\sum_{n} A_{n} E^{\prime \prime} ; x\right) c^{i n K z}
$$

or, introducing the notation $f_{n} \equiv E^{\prime n}(0)$ and $e_{n} \equiv E^{n}(0)$, we have

$$
E_{y}(0, z)=\sum_{n} A_{n} e_{n} e^{i n K z}, E_{y}^{\prime}(0, z)=\sum_{n} A_{n} f_{n} e^{i n K z}
$$

Note that for this problem the plasma surface impedance matrix of Sect. 3 reduces to a scalar surface impedance $Z^{n}=i \omega e_{n} / f_{n}$ such that $E_{y}^{n}(0)=Z^{n} B_{z}^{n}(0)$. We can solve for $A_{n}$ in the first part of Eq. (18) by Fourier transformation,

$$
A_{n}=\frac{1}{b e_{n}} \int_{0}^{b} d z e^{-i n K z} E_{y}(0, z)
$$

which, when used in the second part of Eq. (18), gives

$$
\begin{aligned}
E_{y}^{\prime}(0, z) & =\sum_{n} \frac{f_{n}}{b e_{n}} e^{i n K z} \int_{0}^{b} d z^{\prime} e^{-i n K z^{\prime}} E_{y}\left(0, z^{\prime}\right) \\
& =\int_{0}^{b} d z^{\prime} H\left(z-z^{\prime}\right) E_{y}\left(0, z^{\prime}\right),
\end{aligned}
$$

where the kernel $H\left(z-z^{\prime}\right)$ is given by

$$
H\left(z-z^{\prime}\right)=\sum_{n} \frac{f_{n}}{b e_{n}} e^{i n K\left(z-z^{\prime}\right)} .
$$


Equation (20) is, of course, the reduction to this problem of the more general boundary condition expressed in Eq. (9). If the inte al in Eq. (20) is performed using trapezoidal rule quadrature with increment $h_{z}$, the discretized version of Eq. (20) can be written

$$
E_{y}^{\prime}\left(0, z_{i}\right)=\sum_{j} h_{z} H\left(z_{i}-z_{j}\right) E_{y}\left(0, z_{j}\right)
$$

It is instructive to consider a case in which a single Fourier mode in $z$ is present, $J_{y}(z)=e^{i n K z}$. Then $E_{y}\left(0, z_{i}\right)=E_{0} e^{i n K z_{j}}$ and Eq. (22) can be expressed as

$$
\begin{aligned}
E_{y}^{\prime}\left(0, z_{i}\right) & =h_{z} E_{0} \sum_{j=1}^{N_{z}} H\left(z_{i}-z_{j}\right) e^{i n K z_{j}}=h_{z} E_{0} \sum_{j=1}^{N_{z}} \sum_{m} \frac{f_{m}}{b e_{m}} e^{i m K\left(z_{i}-z_{j}\right)} e^{i n K z_{j}} \\
& =\frac{h_{z}}{b} E_{0} \sum_{m} \frac{f_{m}}{e_{m}} e^{i m K z_{i}} \sum_{j=1}^{N_{z}} e^{2 \pi i\left(j / N_{s}\right)(m-n)} .
\end{aligned}
$$

The second sum is a geometric series that can be evaluated to yield $N_{4} \delta_{m-n, \nu N_{z}}$ for $v-0, \pm 1, \pm 2, \cdots$, so that Eq. (23) reduces to

$$
E_{y}^{\prime}\left(U, z_{i}\right)=E_{0}\left[\frac{f_{n}}{e_{n}} e^{i n K z_{i}}+\frac{f_{n+N_{z}}}{e_{n+N_{z}}} e^{i\left(n+N_{z}\right) K_{i} z_{i}}+\frac{f_{n}-N_{z}}{e_{n}-N_{z}} e^{i\left(n-N_{z}\right) K z_{i}}+\cdots\right] .
$$

The correct value, $E_{y}^{\prime}\left(0, z_{i}\right)=E_{0} f_{n} / e_{n} e^{i n K z_{i}}$, is obtained only if the sum over $m$ in Eq. (23) is restricted to the range $0 \leq|m| \leq N_{z} / 2$ and $n$ itself is restricted to $0 \leq|n| \leq N_{2} / 2-1$. This is the expected result in consideration of the discussion following Eq. $\left(6^{\prime}\right)$.

\subsection{Implementation in a 2D Finite Difference Code}

A finite difference solution of Eq. (15) is to be obtained in the vacuum region $\left(-x_{w} \leq x \leq 0,0 \leq z \leq b\right)$ on the mesh $x_{i}=-x_{w}+i h_{x}, z_{j}=j h_{z}$ for $i=0, N_{x}, j=$ $0, N_{z}$, where $h_{x}=x_{w} / N_{x}, h_{z}=b / N_{z}$. Defining $E_{i, j} \equiv E_{y}\left(x_{i}, z_{j}\right)$, we can write the finite difference form of Eq. (15) as

$$
\frac{1}{h_{x}^{2}}\left(E_{i+1, j}+E_{i-1, j}\right)+\frac{1}{h_{z}^{2}}\left(E_{i, j+1}+E_{i, j-1}\right)+\left[\frac{\omega^{2}}{c^{2}}-2\left(\frac{1}{h_{x}^{2}}+\frac{1}{h_{z}^{2}}\right)\right] E_{i, j}=\frac{-i \omega}{h_{x}} J_{i, j} .
$$


Imposing perfect con tuctivity on the wall gives $E_{0, j}=0$. Periodicity at $z=0, b$ gives $E_{i, 0}=E_{i, v_{z}}$. And continuity of $\partial E_{y} / \partial z$ at $z=0, b$ gives $E_{i, N_{z}+1}=$ $E_{i, 1}, E_{i, 0-1}=E_{i, N_{z}-1}$. Ait the plasma-vacuum boundary, $i=N_{x}$, Eq. (25) requires $E_{N_{x}+1, j}$, which is obtained from the finite difference representation of $E_{y}^{\prime}$

$$
E_{y}^{\prime}\left(0, z_{j}\right)=\frac{1}{2 h_{x}}\left(E_{N_{x}+1, j}-E_{N_{x}-1, j}\right) .
$$

Using Eq. (22) and solving for $E_{N_{x}+1, j}$ gives

$$
E_{N_{z}+1, j}=E_{N_{z}-1, j}+2 h_{x} h_{z} \sum_{j} H_{i, j} E_{N_{z}, j}
$$

where

$$
H_{i, j}=\sum_{m=-N_{z} / 2}^{N_{z} / 2} \frac{f_{m}}{b \varepsilon_{m}} \exp \left[2 \pi i\left(\frac{m}{N_{z}}\right)(i-j)\right] .
$$

Equation (25), with the associated boundary conditions discussed above, constitutes a linear system for $E_{i, j}$ which is solved using a standard matrix inversion package.

\section{RESULTS OF 2D CALCULATION AND DISCUSSION}

The problem sketched in Fig. 2 is 2D only because of the $z$-dependence of the antenna current, the inhomogeneous term of Eq. (15). As a result the problem can easily be solved by Fourier transformation in the $z$-direction. In the plasma region, the field is again given by an expansion of the form of Eq. (16). In the two vacuum regions, region $Y=\left(-x_{w} \leq x<-x_{A}\right)$, region $\mathrm{II}=\left(-x_{A} \leq x \leq 0\right)$, the expansion is of the forma

$$
E_{y}^{\mathrm{I}, \mathrm{II}}(x, z)=\sum_{n} C_{n}^{+} e^{i\left(+\kappa_{n} x+n K z\right)}+C_{n}^{-} e^{i\left(-\kappa_{n} x+n K z\right)},
$$

where $\kappa_{n}$ is the wave number obtained from the vacuum dispersion relation $\left(\omega^{2} / c^{2}\right)=\kappa_{n}^{2}+n^{2} K^{2}$. Imposing the conditions of vanishing $E_{y}$ at $x=-x_{w}$, continuity of the field and its derivative at the plasma-vacuum boundary, and the jump condition $E_{y}^{\prime}\left(-x_{A+}\right)-E_{y}^{\prime}\left(-x_{A-}\right)=-\omega J_{y}$ at the strap location, one can 
eliminate the unknown coefficients $C_{n}^{ \pm}, A_{n}$ and obtain a complete solution. In particular, the expansion coefficients $A_{n}$ in Eq. (16) are given by

$$
A_{n}=-\omega \mu_{0} J_{n} \frac{\sin \left[\kappa_{n}\left(x_{w}-x_{A}\right)\right]}{f_{n} / b_{n} \sin \left(\kappa_{n} x_{w}\right)+i \kappa_{n} \cos \left(\kappa_{n} x_{w}\right)},
$$

where $J_{n}$ is the $n$th Fouricr component of $J_{y}(z)$.

To compare the twa computations we have plotted the field at the plasma boundary $E_{y}(0, z)$ versus $z$ as obtained by the Fourier method of Eq. (28), Fig. 3a, and by the 2D finite difference method, Fig. 3b. The plasma parameters are representative of a modest-size tokamak: $n_{e}(0)=4.5 \times 10^{19} \mathrm{~cm}^{-3}$ with a parabolic profile, $n_{e}(x)=n_{e}(0)\left(1-x^{2} / a^{2}\right), a=60 \mathrm{~cm}, B_{0}=2 \mathrm{~T}$. The cold plasma conductivity was used. Whe geometric parameters were chosen for convenience of comparison of the two calculations: $b=1000 \mathrm{~cm}, x_{A}=5 \mathrm{~cm}$, $x_{w}=15 \mathrm{crn}$. The strap current distribution is constant, with a width of $200 \mathrm{~cm}$, and is centered in the domain, at $z=500 \mathrm{~cm}$. The RF frequency is $f_{\mathrm{RF}}=125 \mathrm{MHz}$. Figure 3a was calculated with 150 Fourier modes. One can see that the field is small except in front of the current strap and that there are oscillations at the ends of the strap due to Gibbs phenomena. The solid curves are $\operatorname{Re}\left\{E_{y}(0, z)\right\}$, and the dashed curves are $\operatorname{Im}\left\{E_{y}(0, z)\right\}$. Figure $3 \mathrm{~b}$ was calculated with 30 grid points in each dimension. The discrete nature of
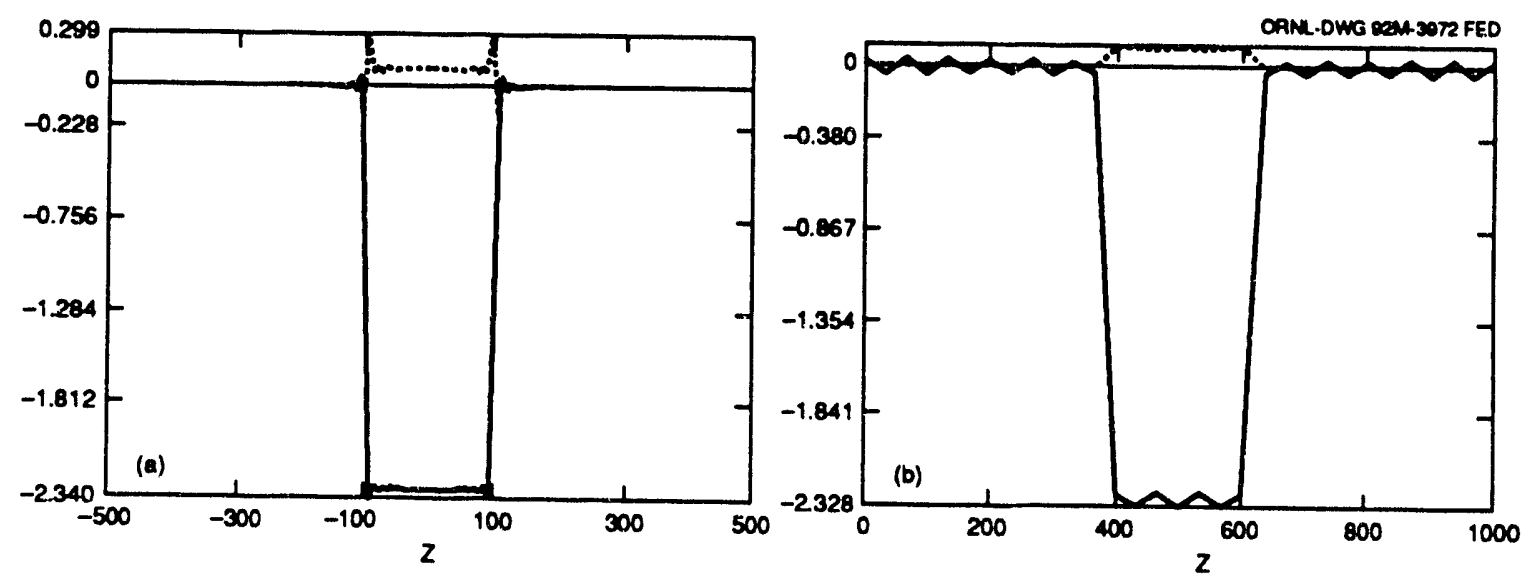

Fig. 3. Electric field at the plasma-vacuum boundary, $\operatorname{Re} E_{y,}(0, z)$ (solid curves) and $\operatorname{Im} E_{y}(0, z)$ (dashed curves), obtained from (a) the Fourier solution and (b) the finite difference solution. 
the solution is evident. The important point is that the field amplitudes agree to within about $1 \%$. The Poynting flux at the first grid point inside the vacuum region obtained from the finite difference solution is shown in Fig. 4. We see that the small-amplitude oscillations appearing in Fig. $3 \mathrm{~b}$ away fron the antenna do not result in a nonphysical power flow there. Another important comparison is the total radiated power in the two models. Summing the power radiated into each mode of the Fourier solution yields $P_{\text {tot }}=0.754 \mathrm{~W} \cdot \mathrm{A}^{-1} \cdot \mathrm{m}^{-1}$. Integrating the real part of $\mathbf{E} \cdot \mathbf{J}$ along the current strap for the finite difference case gives $P_{\text {tot }}=0.736 \mathrm{~W} \cdot \mathrm{A}^{-1} \cdot \mathrm{m}^{-1}$, an agreement of $2 \%$. We conclude therefore that the boundary condition proposed above does indeed yield a well-conditioned linear system and does enforce the proper energy flux on the finite difference solution.

Figure 5 shows a plot of the kerriel function of Eq. (27), $H_{i, 0}$, which is a measure of the coupling between points on the boundary with spacing $\Delta x=i h_{z}$. The function is purely imaginary and highly peaked at $i=0$. This strong coupling of each boundary point to itself and the lack of much interesting structure in $H_{i, 0}$ away from $i=0$ cause one to wonder whether the coupling is in some sense trivial and whether the structure of $H_{i, j}$ is really significant. To

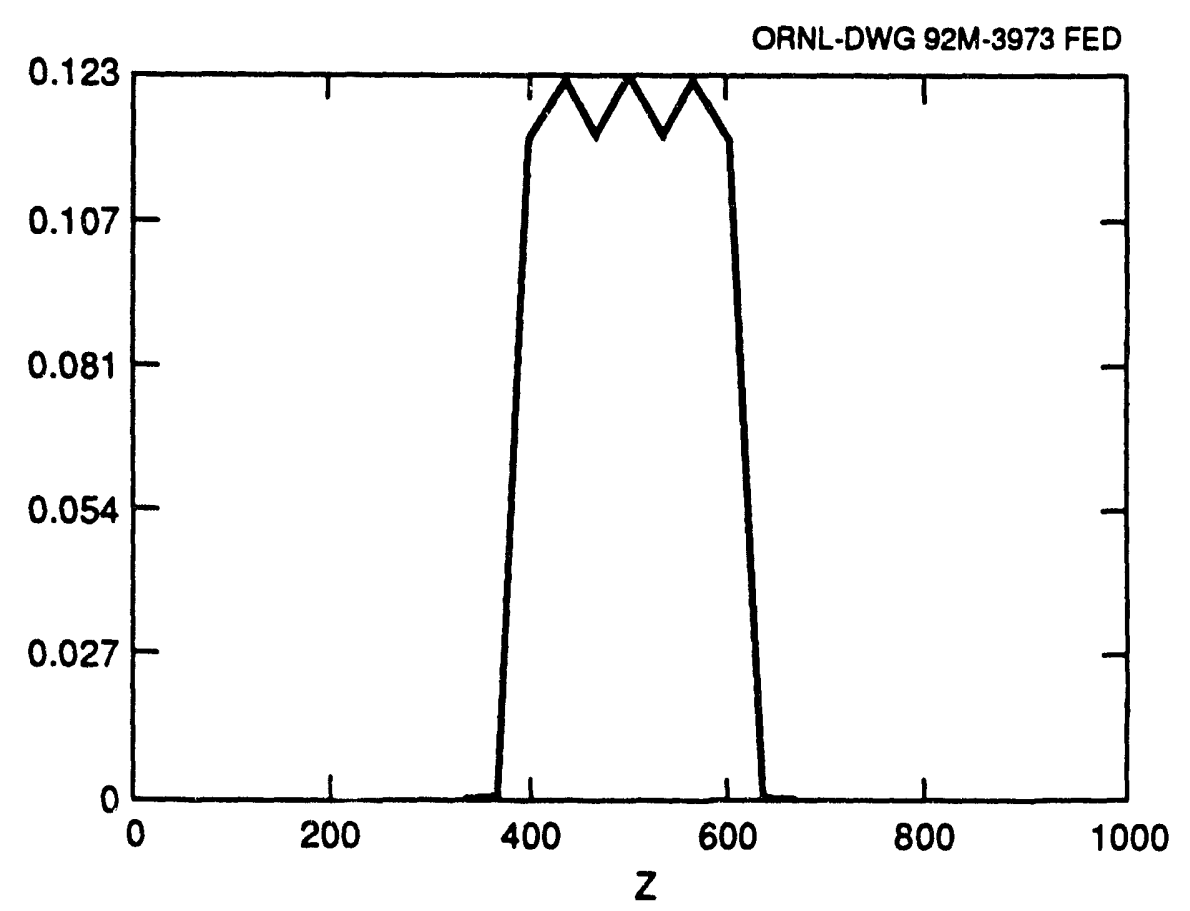

Fig. 4. Poynting flux $S_{x}$ versus $z$ for the finite difference solution (Fig. 3a). 


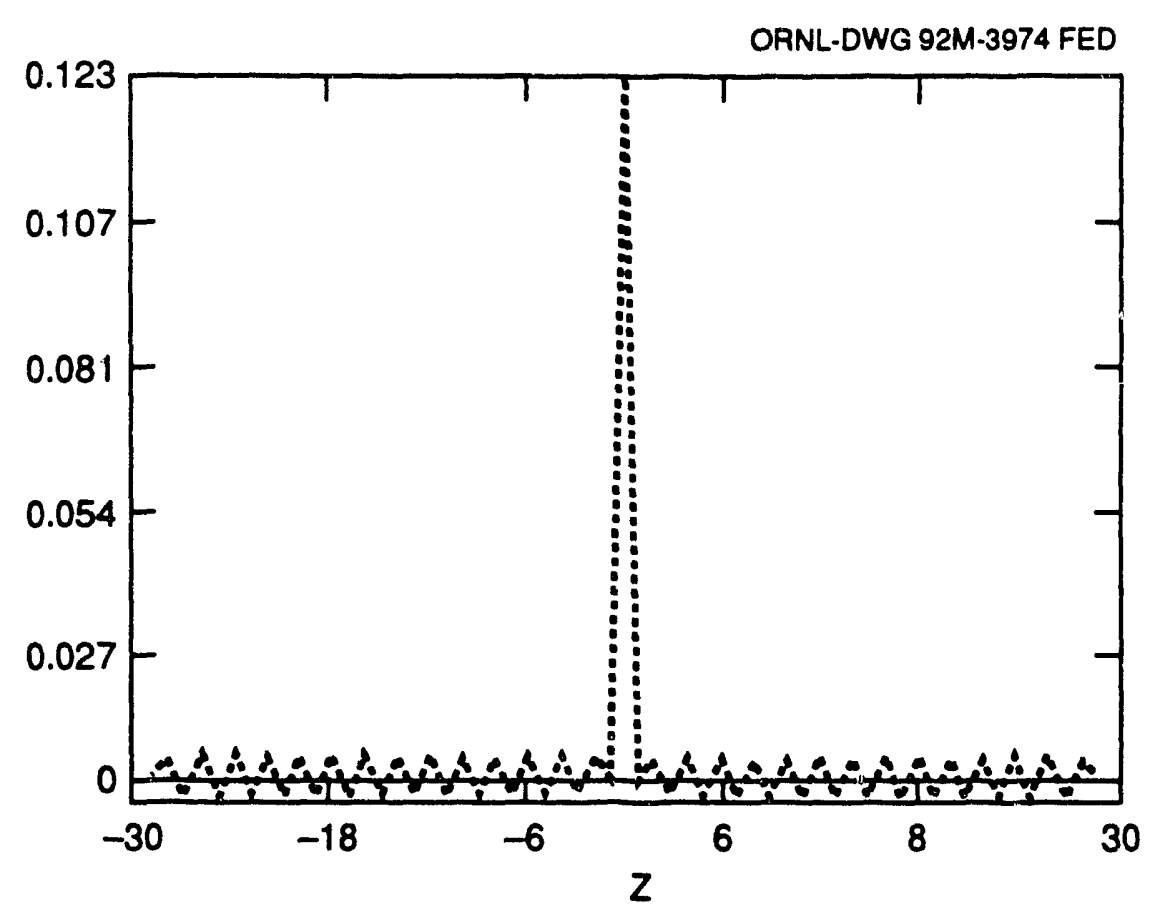

Fig. 5. Kernel function $H_{i, 0}$ versus point spacing, $i$ as calculated for Eq. (27) using the correct number of Fourier modes, $-N_{z} / 2 \leq n \leq N_{z} / 2$.

investigate this, we have run the same cast is hown in Fig. 3 but with the sum in Eq. (27) extending over different ranges of $m$. Figure 6 a shows $H_{i, 0}$ calculated taking one less term at each end of the sum range, $-\left(N_{z} / 2-1\right) \leq n \leq\left(N_{z} / 2-1\right)$. The difference between this and Fig. 5 is rather subtle; however, the effect on the solution, shown in Fig. 6b, is striking compared to Fig. 3b. In this case the imaginary part of the solution is completely unstable, and the calculated power is $P_{\text {tot }}=1.67 \mathrm{~W} \cdot \mathrm{A}^{-1} \cdot \mathrm{m}$, a factor of 2.2 error. Taking an additional term in the sum over $m$ has a smaller but still significant effect.

We anticipate extending this calculation to three dimensions and including all three electric field components. To do this, some computational issues must be resolved. The matrix structure is block-tridiagonal except for the equations connecting to the plasma boundary points, which are dense. Clearly it will not be feasible to solve the complete system arising from the $3 D$ problem as a dense matrix. Since the coupling to non-nearest-neighbor points through the kernel is weak, one could imagine solving the uncoupled problem using sparse matrix techniques, then solving with coupling by iteration. In any case, there are opportunities here for creative linear algebra. 

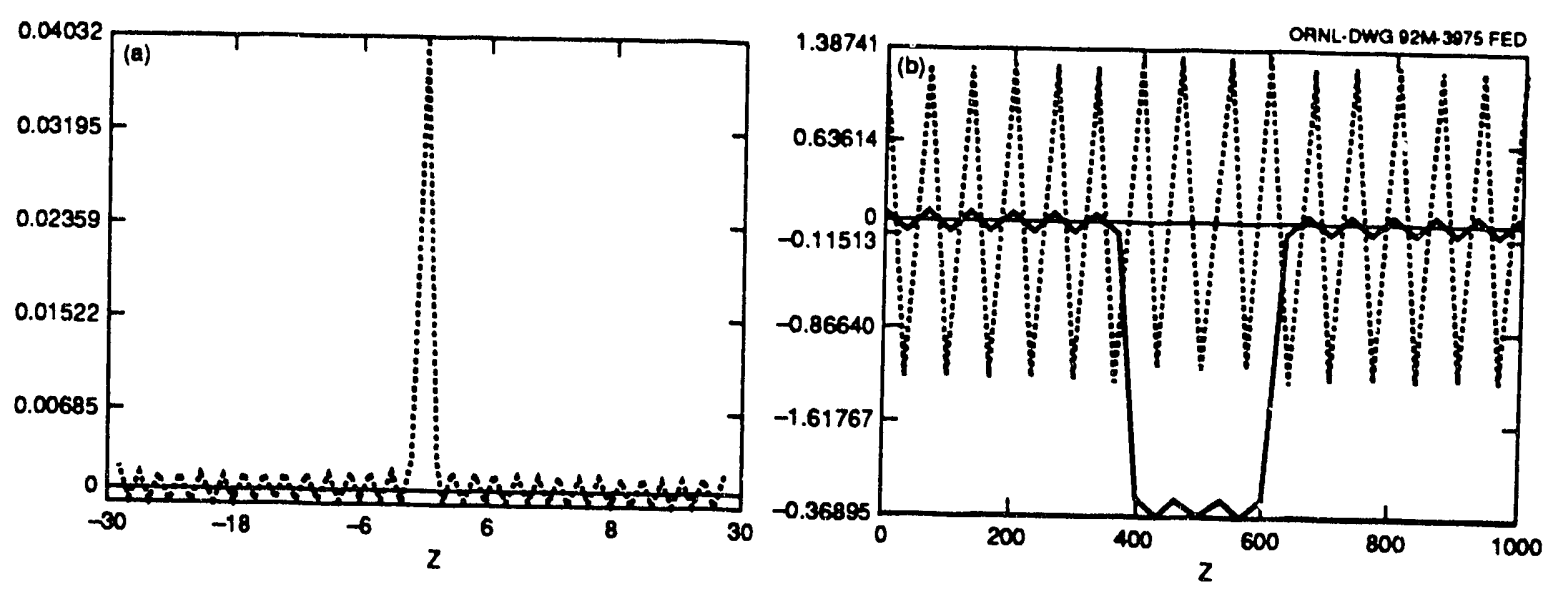

Fig. 6. (a) Kernel function $H_{i, 0}$ versus point spacing, $i$ as calculated for Eq. (27) using fewer Fourier modes, $-\left(N_{z} / 2-1\right) \leq n \leq\left(N_{z} / 2-1\right)$. (b) Electric field at the plasma-vacuum boundary, $\operatorname{Re} E_{y}(0, z)$ (solid curves) and $\operatorname{Im} E_{y}(0, z)$ (dashed curves), obtained from the finite difference solution using the kernel shown in (a).

\section{ACKNOWLEDGMENT}

The authors thank D. C. Stallings, E. F. Jaeger, and Y. L. Ho for useful discussions. 


\section{REFERENCES}

KRESS M., HO Y. L., GROSSMAN W., DOBROTT A., BATCHELOR D. B., RYAN P. M., and CARTER M. D. (1992) AIP Conf. Proc. 244, 213.

RYAN P. M., ROTHE K. E., WHEALTON J. H., and SHEPARD T. D. (1990)

Fusion Eng. Des. 12, 37.

BATCHELOR D. B., CARTER M. D., GOLDFINGER R. C., STALLINGS D. C., and TOLLIVER J. S. (1992) unpublished. 
ORNL/TM-12248

Dist. Category UC-427

\section{INTERNAL DISTRIBUTION}

1. Director, ORNL Fusion Energy Division

2. C. C. Baker

3. M. J. Saltmarsh

4. L. A. Berry

5. B. A. Carreras

6. R. A. Dory

7. J. L. Dunlap

8. H. H. Haselton

9. M. S. Lubell

10. T. E. Shannon

11. R. P. Leinius

12-13. Laboratory Records Department

14. Laboratory Records, ORNL-RC

15-16. Central Research Library

17. Document Reference Section

18. Fusion Energy Division Library

19-20. ET/FE Division Publications Office
21. ORNL Patent Office

22-26. D. B. Batchelor

27-31. M. D. Carter

32. F. W. Baity

33. T. S. Bigelow

34. R. C. Goldfinger

35. R. H. Goulding

36. S. P. Hirshman

37. D. J. Hoffman

38. W. A. Houlberg

39. E. F. Jaeger

40. D. A. Rasmussen

41. P. M. Ryan

42. D. C. Stallings

43. D. W. Swain

44. J. H. Whealton

45. J. J. Yugo

\section{EXTERNAL DISTRIBUTION}

46. Office of the Assistant Manager for Energy Research and Development, U.S. Department of Energy Field Office, Oak Ridge, P.O. Box 2000, Oak Ridge, TN 37831

47. N. A. Davies, Director, Office of Fusion Energy, Office of Energy Research, ER-50 Germantown, U.S. Department of Energy, Washington, DC 20545

48. M. Roberts, Intemational Programs, Office of Fusion Energy, Office of Energy Research, ER-52 Germantown, U.S. Department of Energy, Washington, DC 20545

49. D. E. Baidwin, Lawrence Livermore National Laboratory, P.O. Box 5511, Livermore, CA 94550

50. R. W. Conn, Mechanical, Aerospace, and Nuclear Engineering Department, 6291 Boelter Hall, University of California, Los Angeles, CA 90024-1597

51. P. C. Liewer, MS 138-208, Jet Propulsion Laboratory, 4800 Oak Grove Drive, Pasadena, CA 91109

52. D. Sigmar, Plasma Fusion Center, Massachusetts Institute of Technology, 167 Albany St., NW 16-288, Cambridge, MA 02139

53. K. I. Thomassen, L-637, Lawrence Livermore National Laboratory, P.O. Box 5511, Livermore, CA 94550

54. J. D. Callen, Department of Nuclear Engineering, University of Wisconsin, Madison, WI 53706-1687

55. S. O. Dean, Fusion Power Associates, Inc., 2 Professional Drive, Suite 248, Gaithersburg, MD 20879

56. H. K. Forsen, Bechtel Group, Inc., Research Engineering, P.O. Box 3965, San Francisco, CA 94119

57. R. W. Gould, Department of Applied Physics, California Institute of Technology, Pasadena, CA 91125 
58. R. A. Gross, Plasma Research Laboratory, Columbia University, New York, NY 10027

59. R. J. Hawryluk, Plasma Physics Laboratory, Princeton University, P.O. Box 451, Princeton, NJ 08543

60. D. M. Meade, Plasma Physics Laboratory, Princeton University, P.O. Box 451, Princeton, NJ 08543

61. W. M. Stacey, School of Nuclear Engineering and Health Physics, Georgia Institute of Technology, Atlanta, GA 30332

62. D. Steiner, Nuclear Engineering Department, NES Building, Tibbetts Avenue, Rensselaer Polytechnic Institute, Troy, NY 12181

63. R. Varma, Physical Research Laboratory, Navrangpura, Ahmedabad 380009, India

64. Bibliothek, Max-Planck Institut für Plasmaphysik, Boltzmannstrasse 2, D-8046 Garching, Federal Republic of Germany

65. Bibliothek, Institut für Plasmaphysik, KFA Jülich GmbH, Postfach 1913, D-5170 Jülich, Federal Republic of Germany

66. Bibliothek, KfK Karlsruhe GmbH, Postfach 3640, D-7500 Karlsruhe 1, Federal Republic of Germany

67. Bibliotheque, Centre de Recherches en Physique des Plasmas, Ecole Polytechnique Fédérale de Lausanne, 21 Avenue des Bains, CH-1007 Lausanne, Switzerland

68. R. Aymar, CEN/Cadarache, Departement de Recherches sur la Fusion Contrôlée, F-13108 Saint-Paul-lez-Durance Cedex, France

69. Bibliothèque, CEN/Cadarache, F-13108 Saint-Paul-lez-Durance Cedex, France

70. Library, JET Joint Undertaking, Abingdon, Oxfordshire OX14 3EA, England

71. Library, FOM-Instituut voor Plasmafysica, Rijnhuizen, Edisonbaan 14, 3439 MN Nieuwegein, The Netherlands

72. Library, National Institute for Fusion Science, Chikusa-ku, Nagoya 464-01, Japan

73. Library, International Centre for Theoretical Physics, P.O. Box 586, I-34100 Trieste, Italy

74. Library, Centro Richerche Energia Frascati, C.P. 65, I-00044 Frascati (Roma), Italy

75. Library, Plasma Physics Laboratory, Kyoto University, Gokasho, Uji, Kyoto 611 , Japan

76. Plasma Research Laboratory, Australian National University, P.O. Box 4, Canberra, A.C.T. 2601, Australia

77. Library, Japan Atomic Energy Research Institute, Naka Fusion Research Establishment, 801-1 Mukoyama, Naka-machi, Naka-gun, Ibaraki-ken, Japan

78. G. A. Eliseev, I. V. Kurchatov Institute of Atomic Energy, P.O. Box 3402, 123182 Moscow, Russia

79. V. A. Glukhikh, Scientific-Research Institute of Electro-Physical Apparatus, 188631 St. Petersburg, Russia

80. I. Shpigel, Institute of General Physics, U.S.S.R. Academy of Sciences, Ulitsa Vavilova 38, Moscow, Russia

81. D. D. Ryutov, Institute of Nuclear Physics, Siberian Academy of Sciences, Sovetskaya St. 5, 630090 Novosibirsk, Russia

82. O. Pavlichenko, Kharkov Physical-Technical Institute, Academical St. 1, 310108 Kharkov, Ukraine

83. Deputy Director, Southwestern Institute of Physics, P.O. Box 15, Leshan, Sichuan, China (PRC)

84. Director, The Institute of Plasma Physics, P.O. Box 1126, Hefei, Anhui, China (PRC)

85. R. H. McKnight, Experimental Plasma Research Branch, Division of Development and Technology, Office of Fusion Energy, Office of Energy Research, ER-542 Germantown, U.S. Department of Energy, Washington, DC 20545 
86. D. Crandall, Director, Division of Applied Plasma Physics, Office of Fusion Energy, Office of Energy Research, ER-54 Germantown, U.S. Department of Energy, Washington, DC 20545

87. E. Oktay, Division of Confinement Systems, Office of Fusion Energy, Office of Energy Research, ER-55 Germantown, U.S. Department of Energy, Washington, DC 20545

88. W. Sadowski, Fusion Theory and Computer Services Branch, Division of Applied Plasma Physics, Office of Fusion Energy, Office of Energy Research, ER-541 Germantown, U.S. Department of Energy, Washington, DC 20545

89. R. E. Mickens, Atlanta University, Department of Physics, Atlanta, GA 30314

90. M. N. Rosenbluth, University of California at San Diego, La Jolla, CA 92037

91. D. Schnack, SAIC, 10260 Campus Point Drive, San Diego, CA 92121

92. Duk-In Choi, Department of Physics, Korea Advanced Institute of Science and Technology, P.O. Box 150, Chong Ryang-Ri, Seoul, Korea

93. Library of Physics Department, University of Ioannina, Ioannina, Greece

94. C. De Palo, Library, Associazione EURATOM-ENEA sulla Fusione, CP 65 , I-00044 Frascati (Roma), Italy

95. Laboratorio Associado de Plasma, Instituto Nacional de Pesquisas Espaciais, Caixa Postal 515, 122201, Sao Jose dos Campos, SP, Brazil

96. Theory Department Read File, c/o D. W. Ross, University of Texas, Institute for Fusion Studies, Austin, TX 78712

97. Theory Department Read File, c/o R. Parker, Director, Plasma Fusion Center, NW 16-202, Massachusetts Institute of Technology, Cambridge, MA 02139

98. Theory Department Read File, c/o R. White, Plasma Physics Laboratory, Princeton University, P.O. Box 451, Princeton, NJ 08543

99. Theory Department Read File, c/o L. Kovrizhnykh, Lebedev Institute of Physics, Academy of Sciences, 53 Leninsky Prospect, 117924 Moscow, Russia

100. Theory Department Read File, c/o B. B. Kadomtsev, I. V. Kurchatov Institute of Atomic Energy, P.O. Box 3402, 123182 Moscow, Russia

101. Theory Department Read File, c/o T. Kamimura, National Institute for Fusion Studies, Nagoya 464, Japan

102. Theory Department Read File, c/o E. Maschke, Departemente de Recherches sur la Fusion Controlée, CEN/Cadarache, F-13108 Saint-Paul-lez Durance, France

103. Theory Department Read File, c/o D. Düchs, JET Joint Undertaking, Abingdon, Oxfordshire OX14 3EA, United Kingdom

104. Theory Department Read File, c/o R. Briscoe, Culham Laboratory, Abingdon, Oxfordshire OX14 3DB, United Kingdom

105. Theory Department Read File, c/o D. Biskamp, Max-Planck-Institut fur Plasmaphysik, Boltzmannstrasse 2, D-8046 Garching, Federal Republic of Germany

106. Theory Department Read File, c/o T. Takeda, Japan Atomic Energy Research Institute, Tokai Fusion Research Establishment, Tokai-mura, Naka-gun, Ibaraki-ken, Japan

107. Theory Department Read File, c/o J. Greene, General Atomics, P.O. Box 85608, San Diego, CA 92186

108. Theory Department Read File, c/o R. Cohen, Lawrence Livermore National Laboratory, P.O. Box $5 \$ 11$, Livermore, CA 94550

109. Theory Department Read File, c/o R. Gerwin, CTR Division, Los Alamos National Laboratory, P.O. Box 1663, Los Alamos, NM 87545

110. R. Aamodt, Lodestar Research Corporation, 2400 Central Avenue, Boulder, CO 80301

111. K. Appert, Centre de Recherches en Physique des Plasmas, Ecole Polytechnique Fédérale de Lausanne, 21 Avenue des Bains, CH-1007 Lausanne, Switzerland 
112. M. Ballico, Max-Planck Institut für Plasmaphysik, Boltzmannstrasse 2, D-8046 Garching, Federal Republic of Germany

113. E. Barbato, Associazione EURATOM-ENEA sulla Fusione, Centro Richerche Energia Frascati, C.P. 65, I-00044 Frascati (Roma), Italy

114. A. Bécoulet, Association EURATOM-CEA sur la Fusion Controlée, Centre d'Etudes Nucléaires de Cadarache, B. P. No. 1, F-13108 Saint Paul lez Durances, France

115. S. Bernabei, Plasma Physics Laboratory, Princeton University, P.O. Box 451, Princeton, NJ 08543

116. A. Bers, Plasma Fusion Center, Massachusetts Institute of Technology, 38-260, Cambridge, MA 02139

117. V. Bhatnagar, JET Joint Undertaking, Abingdon, Oxfordshire OX14 3EA, England

118. P. T. Bonoli, Plasma Fusion Center, Massachusetts Institute of Technology, 175 Albany St., NW17-115, Cambridge, MA 02139

119. M. Brambilla, Max-Planck Institut für Plasmaphysik, Boltzmannstrasse 2, D-8046 Garching, Federal Republic of Germany

120. Dr. Vincent Chan, General Atomics, P. O. Box 85608, San Diego, CA 92186

121. D. Ehst, Building 205, Argonne National Laboratory, 9700 S. Cass Avenue, Argonne, IL 60538

122. D. Faulconer, Laboratoire de Physique des Plasmas, EURATOM-Etat Belge, Ecole Royale Militaire, Avenue de la Renaissance 30, 1040 Brussels, Belgium

123. D. J. Gambier, JET Joint Undertaking, Abingdon, Oxfordshire OX14 3EA, England

124. S. N. Golovato, Plasma Fusion Center, Massachusetts Institute of Technology, 175 Albany Street, NW17-113, Cambridge, MA 02139

125. W. Grossman, SAIC, 1710 Goodridge Drive, McLean, VA 22102

126. W. Ho, SAIC, 10260 Campus Point Drive, San Diego, CA 92121

127. J. Hosea, Plasma Physics Laboratory, Princeton University, P.O. Box 451, Princeton, NJ 08543

128. Kwok Ko, Stanford Linear Accelerator Center, P.O. Box 4349, Stanford, CA 94309

129. C. C. Petty, General Atomics, P.O. Box 85608, San Diego, CA 92186

130. C. K. Phillips, Plasma Physics Laboratory, Princeton University, P.O. Box 451, Princeton, NJ 08543

131. M. Porkolab, Plasma Fusion Center, Massachusetts Institute of Technology, 175 Albany Street, NW17-119, Cambridge, MA 02139

132. R. Prater, General Atomics, P.O. Box 85608, San Diego, CA 92186

133. J. Scharer, Electrical and Computer Engineering Department, 1500 Johnson Drive, University of Wisconsin, Madison, WI 53706

134. D. C. Stevens, Courant Institute for Mathematical Sciences, New York University, 251 Mercer Street, New York, NY 10001

135. P. E. Vandenplas, Laboratoire de Physique des Plasmas, EURATOM-Etat Belge, Ecole Royale Militaire, Avenue de la Renaissance 30, 1040 Brussels, Belgium

136. H. Weitzner, Courant Institute for Mathematical Sciences, New York University, 251 Mercer Street, New York, NY 10001

137. J. R. Wilson, Plasma Physics Laboratory, Princeton University, P.O. Box 451, Princeton, NJ 08543

138-85. Given distribution according to OSTI-4500, Magnetic Fusion Energy (Category Distribution UC-427, Theoretical Plasma Physics) 

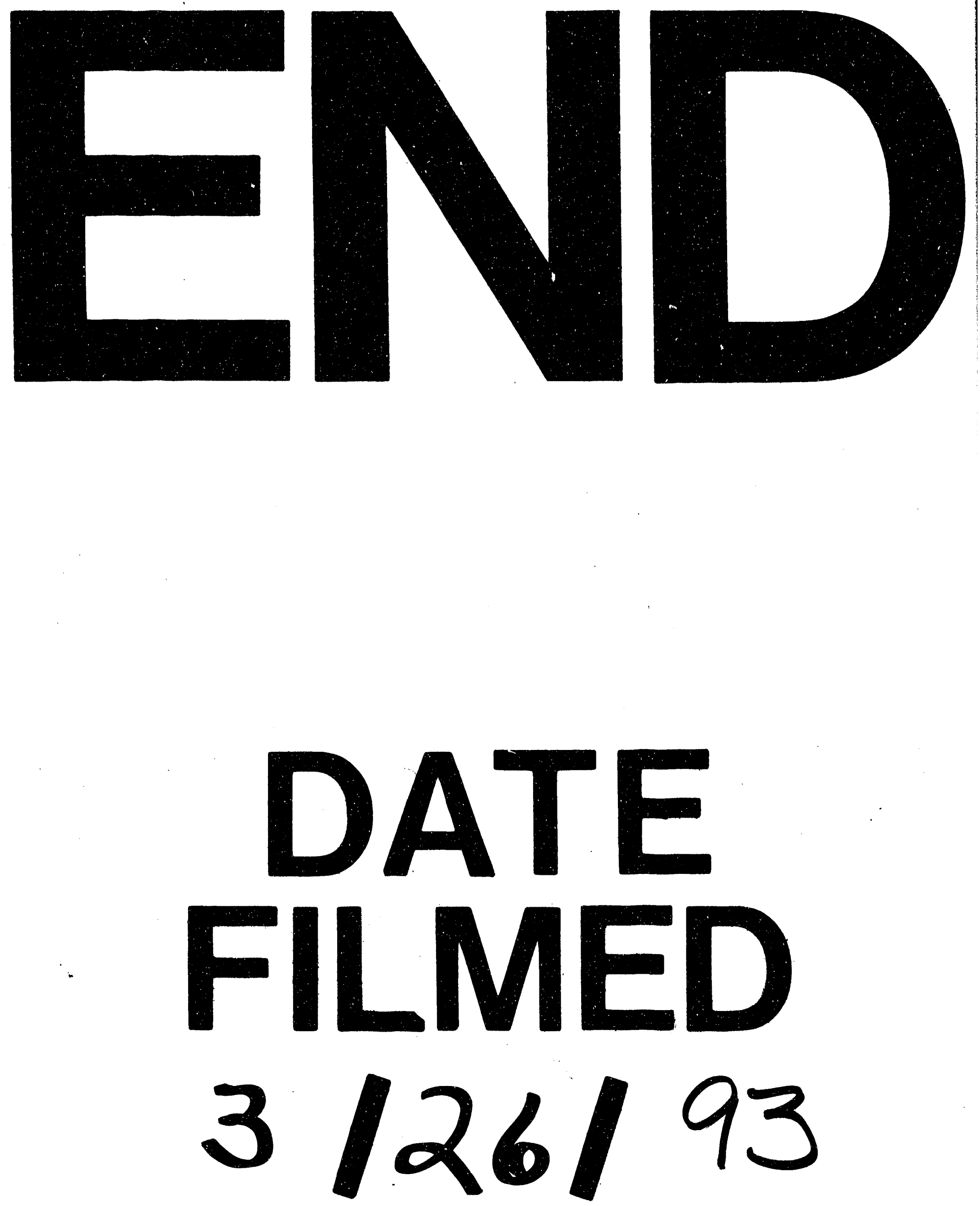
